
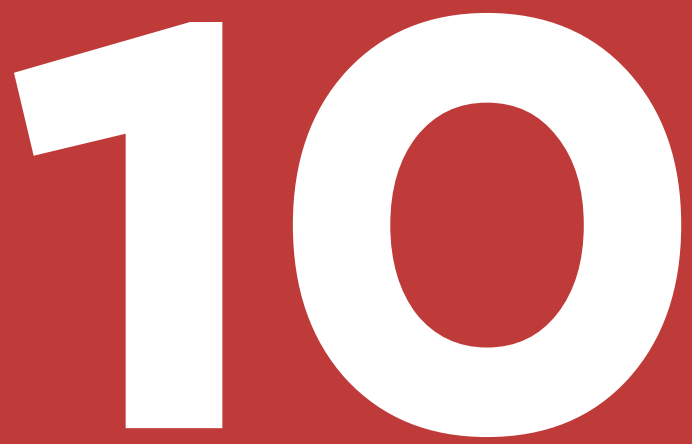

\title{
Fortalecimiento de las capacidades interpersonales de los recicladores de Cuenca
}

Strengthening on interpersonal skills of recyclers in Cuenca

FECHA DE RECEPCIÓN: 28/01/2021

FECHA DE APROBACIÓN:26/07/2021

\section{cc creative \\ c) cointions}




\section{Resumen}

La investigación tuvo como población objetivo a 107 recicladores de las siete asociaciones de Cuenca: Cristo Rey, AREV, El Chorro, ARUC, Feria Libre, Pichacay y San Alfonso. El proyecto fue realizado por profesores y estudiantes de la Escuela de Psicología Organizacional de la Universidad del Azuay. Su principal objetivo fue brindar una alternativa de fortalecimiento dentro del ámbito del comportamiento humano, en competencias tales como el liderazgo, comunicación efectiva, trabajo en equipo, motivación y desarrollo personal, para fomentar la productividad y el desarrollo organizacional. Mediante encuestas, entrevistas a profundidad y grupos focales se realizó un diagnóstico situacional de las relaciones interpersonales de los recicladores, para determinar las oportunidades de mejora, tanto a nivel personal como colectivo. Como principales resultados de este proyecto social, se evidenciaron problemas intrapersonales e interpersonales dentro de sus asociaciones. Para contribuir a mejorar la calidad de vida de esta población vulnerable, se implementaron talleres de capacitación relacionados a la psicología organizacional y adaptada al diagnóstico situacional. El proyecto permitió que los recicladores mejoren sus competencias necesarias para el correcto desenvolvimiento en su ambiente de trabajo y en sus hogares. Asimismo, fue una gran oportunidad para que los estudiantes participen activamente en las necesidades de la comunidad.

Palabras clave: comportamiento de grupo, desarrollo de competencias, psicología organizacional, recicladores.

1 Universidad del Azuay ·jdbravo@uazuay.edu.ec • https://orcid.org/0000-0003-3732-5151 •

Cuenca, Ecuador 


\section{Abstract}

he present research work had a target population of 107 recyclers from the seven associations in the city of Cuenca: Cristo Rey, AREV, El Chorro, Feria Libre, Pichacay y San Alfonso. This project was realized by teachers and students of the Organizational Psychology's school of the Universidad del Azuay and had as its main purpose offer to waste pickers with a strengthening alternative within the field of human behavior, in skills such as leadership, effective communication, teamwork, motivation and personal development; through them, promote productivity and organizational development into the associations they are part of. Through surveys, in-depth interviews and focus groups, a situational diagnosis of the interpersonal relationships of each association was carried out in order to determine opportunities for improvement, both personally and collectively level. As main results of this project of engagement with society, intrapersonal problems were evidenced in the recyclers, as well as interpersonal problems within associations. Looking to contribute for improving the quality of life of this vulnerable group, training organizational workshops were elaborated and implemented, adapting them to the situational diagnosis. The project allowed people to improve their abilities and skills necessary for the proper development in their work environment and in their homes. It was also a great opportunity for students to form by participating and actively serving in the community needs.

Keywords: group behavior, training, recyclers, skills development, work psychology.
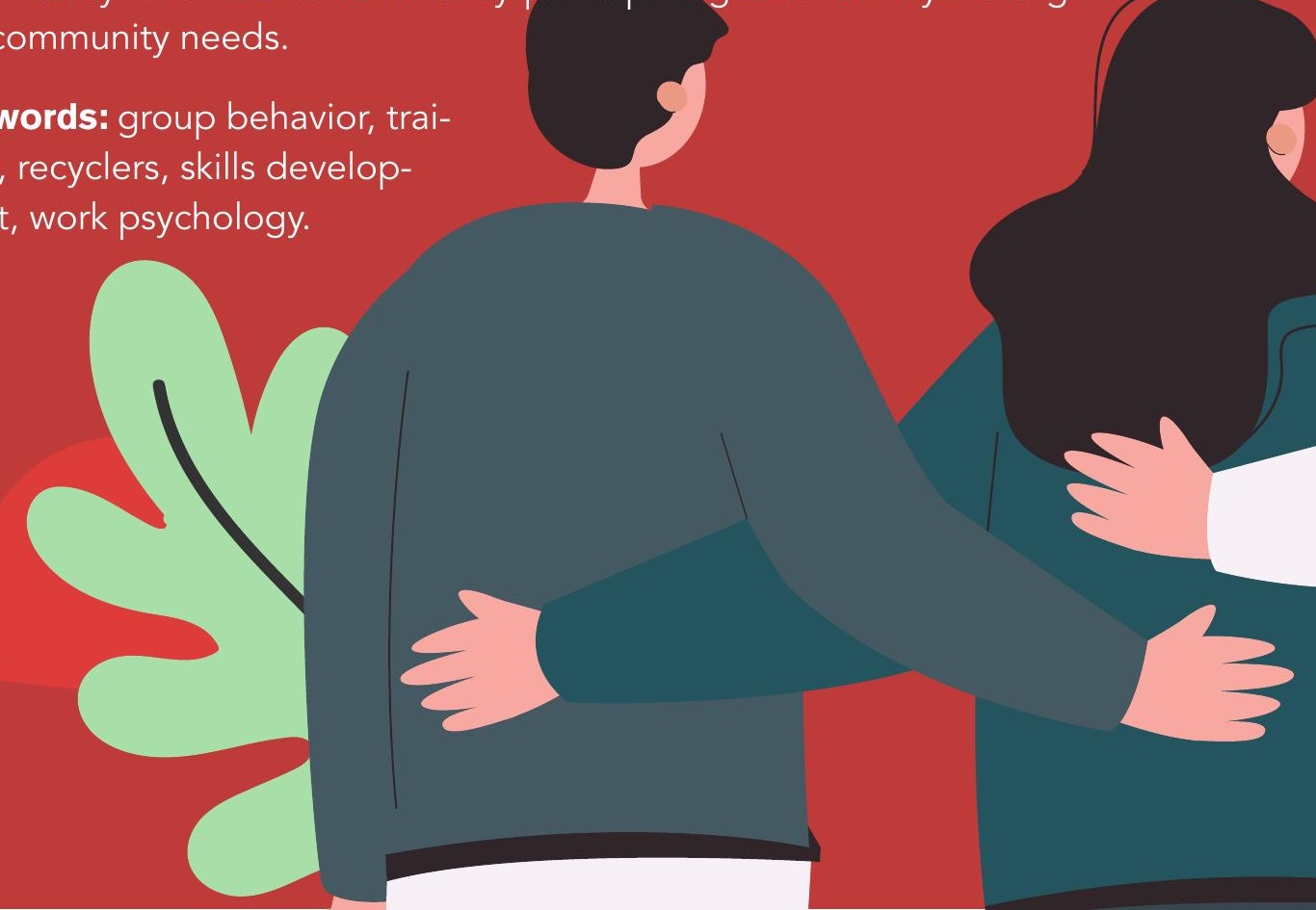


\section{Introducción}

I desarrollo de capacidades interpersonales es uno de los factores cruciales para alcanzar un aumento de la productividad laboral y un mejoramiento del bienestar personal. Las personas, por naturaleza, son seres gregarios que desde el inicio de sus vidas están en constante intercambio con su entorno. Para ello, es importante que se desarrollen ciertas capacidades y habilidades que permitan una adecuada interacción con los demás, potencializando sus capacidades y respetando sus diferencias. Ante ello, es importante resaltar que dicha interacción no es estar por encima de las personas, sino lograr satisfacción equilibrada en la resolución de diferencias existentes en un momento determinado (Sánchez, Magdaniel, \& Sánchez, 2013).

Por ello, es trascendental, en toda organización, que las personas desarrollen ciertas habilidades sociales que les permita, sobre todo, gestionar adecuadamente sus pensamientos y emociones. A esta habilidad se la conoce como inteligencia emocional, la que, a su vez, está formada por dos componentes: por una parte, están las relaciones interpersonales, las cuales, se refieren a la manera en la que interactuamos con las demás personas de nuestro medio; mientras que, por otro lado, está la inteligencia intrapersonal, que según Campbell y Dickinson (2002) la definen como "la capacidad de una persona para construir una percepción precisa respecto de sí misma y utiliza dicho conocimiento para organizar y dirigir la propia vida".

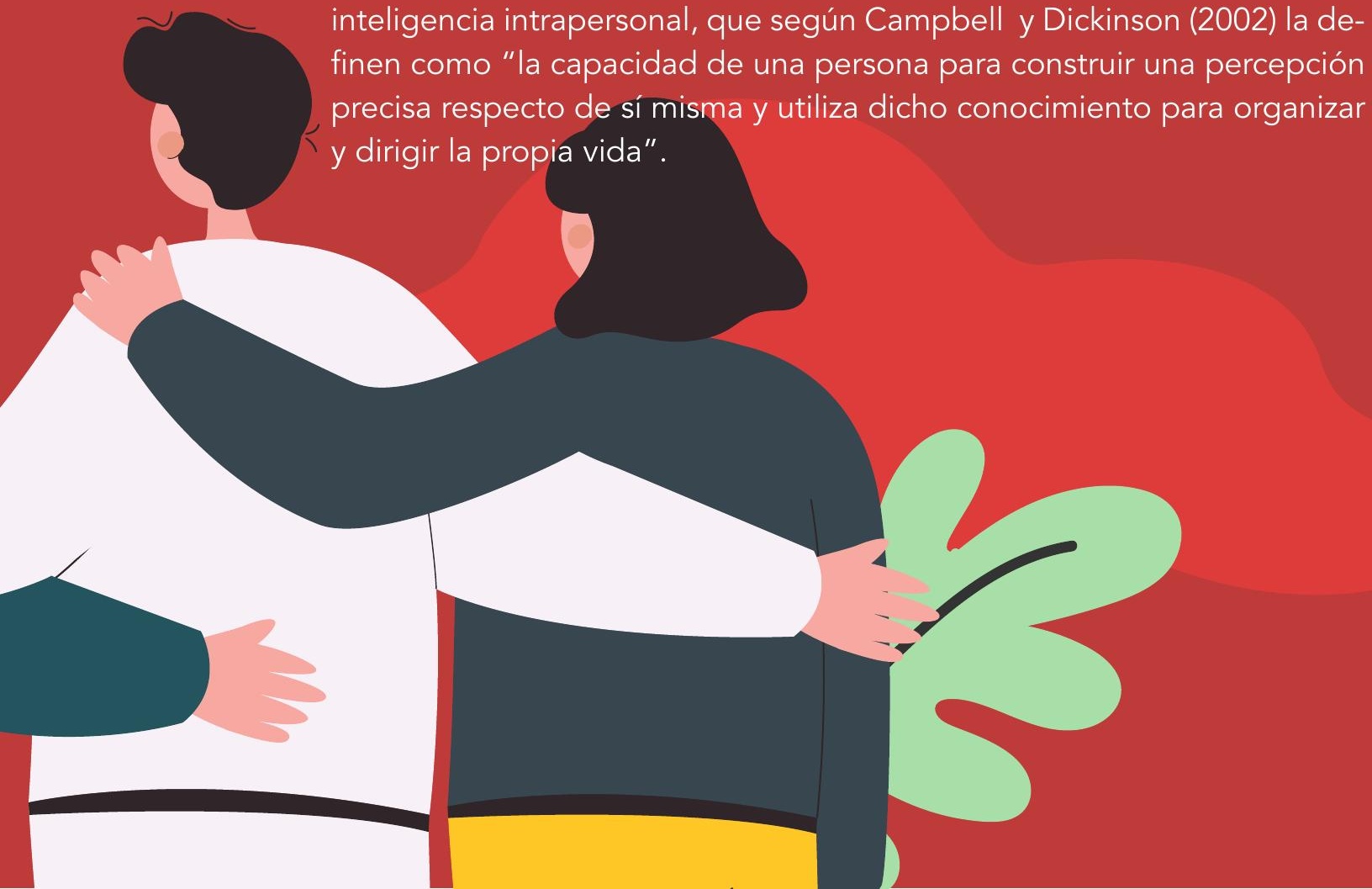


Las emociones funcionan como un coreógrafo organizador de respuestas dispares. Organiza la acción de múltiples respuestas, para actuar de manera unificada al servicio de la resolución de problemas fundamentales (Levenson, 1999). La persona no nace con un repertorio determinado de habilidades sociales, sino que va incorporándose a lo largo de su desarrollo, formación y crecimiento. Hay dos tipos de habilidades sociales: básicas y complejas, siendo necesario el aprendizaje de las primeras para desarrollar las segundas (Salavera \& Usán, 2018).

Cornejo y Tapia (2012) hacen un aporte valioso en cuanto a las relaciones interpersonales. Mencionan que se da una interacción recíproca entre las personas, la cual, involucra destrezas sociales y emocionales que incluye comunicarse efectivamente, escucharse, solucionar conflictos y expresarse. A pesar del valor que tienen las relaciones interpersonales, en las organizaciones se suele ignorar su importancia, lo que se traduce en falta de oportunidades para desarrollarlas. Las relaciones interpersonales positivas contribuyen a la satisfacción laboral, por lo que cada vez es más relevante su rol entre los pares en el trabajo; estas relaciones se basan, principalmente, en la confianza mutua de los miembros de las organizaciones (Yánez, Arenas, \& Ripoll, 2010).

En este sentido, los recicladores asociados de la ciudad de Cuenca, quienes realizan una actividad muy importante para la convivencia social y para la conservación del medio ambiente, no han tenido la oportunidad de fortalecer esas competencias, debido a la vulnerabilidad que conlleva su trabajo y su condición socioeconómica. La exclusión social rompe las relaciones que tenemos con otros e influencia nuestra salud física y mental en una amplia gama de formas (Kawamoto, Ura, \& Nittono, 2015).

Si tomamos como referencia la Constitución de la República del Ecuador (2008), en su artículo 66, habla de que las personas tienen derecho a la integridad psíquica así como al desarrollo de la personalidad y que el Estado adoptará medidas necesarias para prevenir toda forma de violencia, en especial, contra personas en situación de desventaja o vulnerabilidad. En correspondencia con esto, el Plan Nacional de DesarroIlo 2017-2021 (SENPLADES, 2017), en su objetivo uno habla de que las instituciones públicas, de manera obligatoria; y, los sectores privados, de manera indicativa, deberán lograr una vida digna para todas las personas, en especial, aquellas en situación de vulnerabilidad, incluye la pro- 
moción de un desarrollo inclusivo que empodere a las personas durante todo el ciclo de vida, mediante educación y capacitación de calidad y pertinente. Esto, con el fin de potenciar las capacidades y el talento de las personas, concibiendo a la educación desde una mirada humanista del aprendizaje a lo largo de toda la vida, con miras al desarrollo social, económico y cultural.

A pesar de que las siete asociaciones de recicladores de Cuenca son apoyadas en algún aspecto por instancias gubernamentales, sus 107 integrantes carecen de oportunidades de fortalecimiento y reflexión sobre sus conductas al interior de las organizaciones y cómo estas contribuyen a la mejora o detrimento de sus relaciones laborales y personales.

Al ser asociaciones independientes con objetivos comunes, surgen inconvenientes en cuanto a la disputa por obtener mayor cantidad de material reciclable; esto quiere decir que no siempre existe una comunicación efectiva dentro y fuera de los grupos. Esto, sin duda, afecta el correcto desempeño de sus labores diarias; es decir, al no reconocer sus necesidades y sentimientos ni hacerlas saber con confianza y claridad, se da la falta de respeto y la mala intención hacia los demás (Naranjo, 2011). Adicionalmente, hay falta de organización en las tareas asignadas, ya que muchos se quejan de que no siempre les alcanza el tiempo para realizar todo el trabajo.

Por estas y otras razones, fue importante que en este proyecto exista una participación activa de la población objetivo, ya que su valiosa experiencia, combinada con las teorías psicológicas y organizacionales, contribuye de manera más efectiva al desarrollo del proceso de fortalecimiento de las capacidades (Balcazar, 2003).

Las personas son el blanco preferido del empoderamiento social, con frecuencia entendido como autoestima, autoeficacia o competencia personal. La autoestima se construye dependiendo de la aceptación que tengamos en el medio en el cual nos desenvolvemos, de cómo nos reciben los demás; ya que como dice Naranjo (2007), la autoestima se desarrolla a partir de la interacción humana, mediante la cual las personas se consideran importantes una para las otras. El yo evoluciona por medio de pequeños logros, los reconocimientos y el éxito. 
A la inteligencia intrapersonal se la puede definir como la capacidad que nos permite conocernos mediante un autoanálisis; es la capacidad de conocerse a sí mismo y actuar en consecuencia. Esta se refiere a la autocomprensión, el acceso a la propia vida emocional, a la propia gama de sentimientos, a la capacidad de efectuar discriminaciones de estas emociones $y$, finalmente, ponerles nombre y recurrir a ellas como medio de interpretar y orientar la propia conducta. Desde el punto de vista personal, el sentimiento es lo primero que debe explicarse, la causa última de los comportamientos considerados emocionales y lo que hay que cambiar cuando uno quiere regular la emoción (Campos, Dahl, Walle, \& Main, 2011).

Al tener desarrollada la inteligencia intrapersonal, mantenemos la capacidad de ver con realismo y veracidad, qué somos y qué queremos, estableciendo prioridades y anhelos personales para, de esta forma, actuar en consecuencia. Las personas con este tipo de inteligencia desarrollada, no suelen engañarse con respecto a sus propios sentimientos y emociones y saben cómo respetarlos, siendo capaces de analizar el porqué de sus pensamientos, de sus actitudes y corregirlos. En la actualidad, las relaciones interpersonales se hallan sometidas a singularidades, presiones y simbolismos que las caracterizan, máxime ante unas condiciones relacionales de cambios en los estilos de expresión de los afectos y sentimientos; de ahí, la conveniencia de evaluar de forma integral estas dependencias (Freitas-Magalhaes, 2007).

Para lograr el equilibrio emocional es fundamental conocer cómo satisfacer nuestras necesidades emocionales, lo que nos permite calmarnos ante situaciones estresantes y actuar con practicidad y eficacia, evitando que tengamos reacciones desmedidas ante determinadas situaciones. Con la regulación integrada, las personas tienen la plena sensación de que el comportamiento es una parte integral de quienes son, que emana de su sentido de sí mismo y, por lo tanto, está autodeterminado (Gagne \& Deci, 2005). Todo esto redunda en la consecución de un marcado bienestar emocional que influye positivamente en el resto de inteligencias, así como en el plano físico.

Una persona con baja autoestima se siente desadaptada, piensa que vale poco, le cuesta trabajo tomar decisiones importantes por no confiar en su propias habilidades. Una persona con alta autoestima está más motivada a hacia la autorrealización, pues, genera sustancias y ac- 
tiva procesos internos que influyen de manera directa en su actuación (Estrada, 2018). La adquisición de poder personal puede ser el punto de partida para que las personas se embarquen en actuaciones que, si son exitosas, generan poder objetivo, a menudo, colectivo (Sánchez-Vidal, 2017).

Es importante señalar que la condición de vulnerabilidad de esta población debe ser abordada desde una base de prioridades bien reconocidas para el bienestar humano, que incluyen la seguridad alimentaria,

los ingresos, agua y saneamiento, salud, educación, energía, igualdad de género, equidad social y puestos de trabajo (Velenturf \& Jopson, 2019). En este caso, al hablar

de salud, consideramos la integralidad enfocándonos, sobre todo, en la salud psicológica que promueve la salud física: Mente sana en cuerpo sano. 


\section{Metodología}

Para el desarrollo del proyecto se utilizó un enfoque mixto (cuantitativo y cualitativo) con un alcance descriptivo, ya que el objetivo fue analizar y contextualizar una situación para luego determinar el impacto de las intervenciones realizadas. Desde el diagnóstico hasta la implementación del proyecto, se utilizó la técnica de la investigación acción participativa, para comprender la realidad de los actores sociales $y$, juntamente con ellos, construir las alternativas que respondan a sus principales problemas psicológico-organizacionales. En este contexto, se participó de las asambleas efectuadas el último martes de cada mes, en la planta de operaciones de la Empresa Municipal de Aseo de Cuenca - EMAC; adicionalmente, hubo visitas por varias ocasiones en sus respectivas plantas recicladoras.

El universo de investigación fueron las siete asociaciones de recicladores de Cuenca: Cristo Rey, AREV, El Chorro, ARUC, Feria Libre, Pichacay y San Alfonso; dentro de estas asociaciones laboran 107 personas, en su mayoría mujeres.

En el enfoque cuantitativo se realizaron encuestas a todo el universo de la población, con el objetivo de encontrar factores coincidentes dentro de la interrelación en las asociaciones. Por otro lado, bajo el enfoque cualitativo, se realizaron entrevistas a profundidad, las cuales, sirvieron para ahondar en los aspectos psicológicos internos de cada persona, así como en su percepción acerca de las formas de comportamiento respecto a los grupos y cómo afectan positiva o negativamente en la dinámica organizacional.

En cuanto a la evaluación de impacto, se definieron previamente indicadores cualitativos que fueron evaluados mediante grupos focales. Esto permitió analizar el resultado de las intervenciones respecto al mejoramiento de las relaciones interpersonales, automotivación, liderazgo y organización interna. 


\section{Resultados}

Desarrollar competencias intrapersonales e interpersonales en los recicladores asociados de la ciudad de Cuenca ha sido un reto, debido a que en su mayoría son personas con limitadas posibilidades de acceder a servicios como formación y capacitación en temas de motivación, comunicación efectiva, habilidades sociales, trabajo en equipo, desarrollo organizacional y otras competencias que hoy en día se consideran claves para el correcto desempeño en las organizaciones y en la vida personal.

La figura del reciclador, generalmente, asocia la escena de grupos de personas recogiendo basura en basureros a cielo abierto o guiando su carreta disputándose lugar a los autos de las grandes ciudades (Diaz-Cano, 2018). A esto hay que sumarle cierta desmotivación, producto del poco nivel de ingresos que generan (muchos recicladores tienen otras actividades económicas), inconvenientes administrativos o políticos con algunas instituciones del Estado y la falta de compromiso de la ciudadanía, sobre todo, en el manejo y clasificación de los desechos. Sin embargo, estos aspectos escapan del giro de esta investigación, por lo tanto, el estudio se centrará en los aspectos psicológicos de cada uno y en sus relaciones interpersonales.

Los aspectos psicológicos son los que, en ciertas ocasiones, impiden que un grupo funcione eficientemente. David McClelland (1973), en su investigación acerca de las competencias laborales, detectó que en las personas existen ciertas características que predicen el éxito. Estas características, desarrolladas y gestionadas correctamente dentro de los grupos de trabajo, pueden llevar a elevar el desempeño organizacional. Asimismo, este autor plantea que la inteligencia intelectual no es el único predictor de éxito laboral. Muchas veces es más importante la forma en cómo se relacionan las personas con sus jefes, usuarios y miembros de equipo, es decir, la inteligencia emocional. Y la mejor forma de relacionamiento es la comunicación asertiva, cuya importancia radica en que es una conducta y no una característica de la personalidad, por lo que se habla de una habilidad en el campo de las habilidades sociales, susceptibles de entrenamiento (Rodríguez, 2019). 
Trabajar con un grupo vulnerable de bajos recursos, otorgándoles capacidades y destrezas que les permita desarrollarse en el ámbito personal, favorece el cumplimiento del Objetivo de Desarrollo Sostenible 3, el cual, hace mención a la salud y bienestar, entendida esta como salud física y mental. (PNUD, 2020).

Con el objetivo de conocer cómo estaban las relaciones intrapersonales e interpersonales de los recicladores, se realizaron 107 encuestas estructuradas, cuyos resultados e interpretación se presenta a continuación: 

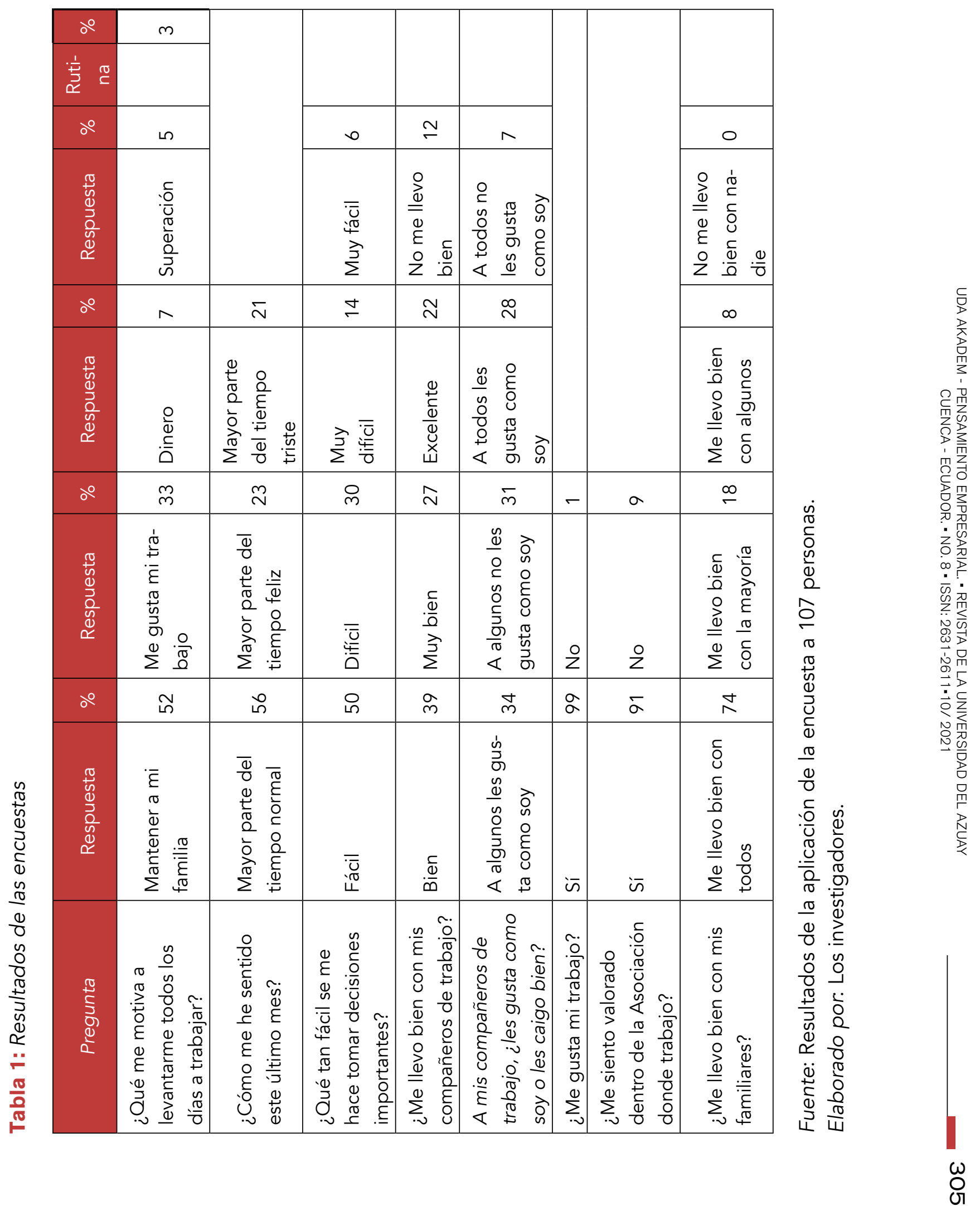
Como producto de las encuestas se puede evidenciar, a nivel general, que los recicladores gustan de su trabajo, así como lo consideran una importante fuente de sustento para su familia, lo que resulta un motivante, tanto intrínseco como extrínseco. Por otro lado, el estado de ánimo de los recicladores ha permanecido mayormente normal, a pesar de que un porcentaje del $23 \%$, manifestó haberse sentido feliz; y, un $21 \%$, triste.

Para los recicladores, la toma de decisiones no es tarea sencilla, pues solo la mitad ha manifestado que les es fácil hacerlo. Esto hace suponer que dentro de las asociaciones podría haber factores laborales o personales que dificultan llegar a acuerdos a la hora de decidir alguna tarea que involucre a todos.

En general, los recicladores se llevan bien entre ellos. Solo el 12\% afirma no llevarse con nadie, lo cual, no es muy significativo, pero, puede ser una alerta a considerar. Este resultado contrasta con la apreciación de cómo los perciben los demás. Alrededor del $38 \%$ de los recicladores perciben que no caen bien al grupo de trabajo, lo cual, tiene un trasfondo de percepción que debería ser contrapuesto con una intervención a nivel grupal. Algo muy importante a destacar es que la gran mayoría de recicladores se sienten valorados dentro de la asociación a la cual pertenecen. Esto es una ventaja organizacional y debe ser alimentada permanentemente para que se desarrolle y no decaiga.

Por último y no menos importante, está la relación que tienen los recicladores en sus núcleos familiares, ya que detrás de un reciclador está un padre o madre de familia, un hijo, un hermano, un abuelo, etc. Se pudo evidenciar que las relaciones personales del grupo no presentan inconvenientes de relevancia; sin embargo, cabe recalcar que la intervención a nivel organizacional está ligada a un cambio de conducta en todos los ámbitos, por lo que las personas se ven beneficiadas, tanto en su trabajo como en su hogar.

Adicionalmente a las encuestas, se realizaron entrevistas a los representantes de cinco asociaciones de recicladores, cuyo rol de liderazgo dentro de la organización ayudó a validar la información inicial. Las entrevistas constituyeron un complemento a la información cuantitativa, las cuales, proyectaron información de carácter cualitativo que, para me- 
jor comprensión, se la agrupó y sintetizó en tres categorías: inteligencia emocional, autoestima y desarrollo interpersonal e intrapersonal.

\section{a) Inteligencia emocional}

La inteligencia emocional se refiere a la manera en la que uno expresa sus emociones y la forma de apreciar las emociones de los demás. Fernández \& Extremera (2005) hablan de la facilitación o asimilación emocional, la cual, implica la habilidad para tener en cuenta los sentimientos, cuando razonamos o solucionamos problemas. Esta habilidad se centra en cómo las emociones afectan al sistema cognitivo y cómo nuestros estados afectivos ayudan a la toma de decisiones.

Es por esto que en las preguntas referidas a este aspecto, se presentan distintas situaciones en donde las personas, de acuerdo a las experiencias vividas, buscaron la manera de manejar efectivamente sus emociones, reconociendo así aspectos tanto positivos como negativos y la forma en la que un problema con terceras personas se transformó en una relación positiva. Por ejemplo, uno de los entrevistados comenta que cuando viaja para traer material, se siente satisfecho cuando cumple con lo solicitado, frente a las emociones negativas que experimenta cuando sus horas de trabajo son superiores a las establecidas a más de existir problemas con sus compañeros de trabajo; sin embargo, menciona que el diálogo es uno de los principales recursos para solucionar estas discrepancias, permitiéndoles llegar a acuerdos mutuos.

Otra situación que les ha permitido apreciar sus emociones buenas y malas es en aspectos referentes al dinero; sienten que la cantidad de material reciclado no representa el dinero que reciben por la venta, en este caso, se han tomado medidas en las que explican a los trabajadores que el dinero está bien manejado y no existen los inconvenientes que ellos suponen.

La inteligencia emocional no se queda solo en el entender o comprender, sino en respetar lo que el otro siente y decide; esto se ve reflejado en la entrevista que describe la situación en la que los recolectores querían llevarse todo lo reciclado y la gerente tuvo que dirigirse a ellos expresando que esto no era posible, contribuyendo así a una mejor convivencia entre los involucrados. 
Las personas pueden vivir situaciones similares, pero la forma de reaccionar va a depender de su inteligencia emocional, es decir, a la capacidad de sustituir las emociones negativas por positivas y buscar el camino adecuado para solucionar los conflictos que se les presenta a diario.

\section{b) Autoestima}

La autoestima es la evaluación global que una persona hace de sí misma y los sentimientos que acompañan dicha evaluación (Werkuyten \& Nekuee, 2001). Es el valor que el individuo otorga a su persona y a sus capacidades. Es pensar positivamente para enfrentar retos que implican que nosotros nos aceptemos, respetemos, confiemos y creamos en nosotros mismos. La valoración de uno mismo se basa en todos los sentimientos y experiencias por las que pasamos y hemos recogido durante nuestra vida.

Según las experiencias de los representantes en convivencia con sus equipos de trabajo, las emociones al interior cambian constantemente, en mayor medida, por la escasez de recursos, lo cual, hace que se sienten desvalorizados, sobre todo, cuando la paga no es a tiempo. Aquí es cuando los trabajadores empiezan a tener quejas en contra de ellos. Al parecer la actividad económica que mantienen es un gran factor para su autoestima, porque no se sienten apreciados y retribuidos cuando realizan el mejor trabajo posible.

Reconocen que la autoestima es un punto clave para el éxito o el fracaso; por ello, procuran motivar y reconocer la importancia del trabajo de cada miembro de la asociación con palabras, bromas, anécdotas y demás momentos de interacción personal. A su vez, consideran que deben generar mayor confianza e interés por el desarrollo de los trabajadores, para que exista respeto y aceptación por uno mismo. Esto lo lograrán en la medida que cuenten con todos los recursos materiales, no solo para realizar su trabajo sino para poder corresponder el esfuerzo diario de cada reciclador. 


\section{c) Desarrollo interpersonal e intrapersonal}

Las personas son científicos, tratando de entenderse a sí mismos, a su ambiente y, luego, actuando sobre la base de este conocimiento (Weigner, 1992). El proceso de establecer relaciones interpersonales e intrapersonales, guarda una relación muy estrecha con la manera de expresar las emociones, los sentimientos y la forma de plantear las ideas. Cuando las personas no actúan sobre la base de la tolerancia, respeto y armonía, originan relaciones caracterizadas por la agresividad y frustración, lo que ocasiona, entre otras cosas: discordia, desdenes, amenazas; relaciones sociales conflictivas que casi siempre violentan los derechos de las personas y generan conflictos (Suárez \& Mendoza, 2008).

Centrando los resultados en el ámbito interpersonal se puede ver en los trabajadores una amplia capacidad en el "darse cuenta" para poder afrontar sus errores y mejorar sus capacidades de respuesta, teniendo una conciencia de sí mismos y de las situaciones por las que están pasando y, al final, analizar cuál es el mejor recurso para afrontarlo.

La percepción de los representantes de los grupos ante estas categorías es que los recicladores buscan en sí mismos un refugio emocional ante las situaciones por las que les toca pasar. La labor del reciclador es dura y compleja, por lo que tratan de ser un apoyo el uno para el otro, incluso, para desfogarse de la pesada carga que tienen en sus hombros.

\section{Impacto}

Nagles (2005) en su artículo: El desarrollo de competencias, señala que las organizaciones requieren atender, en forma efectiva y con gran velocidad, los desafíos que se generan en el ambiente donde se desarrollan las diferentes actividades. Una forma de generar respuestas efectivas a esos desafíos es preparar y ayudar a las personas para que desarrollen las competencias necesarias, con el fin de asegurar un buen desempeño personal y profesional. La motivación juega un rol fundamental, ya que genera innovación y creatividad, a través de cualquier influencia social o cultural del comportamiento en el trabajo (Steers \& Mowday, 2004).

En un segundo momento, se procedió a evaluar el impacto de las intervenciones realizadas con los recicladores, tanto en el ámbito perso- 
nal como ocupacional. Se realizaron diez entrevistas al azar entre todas las asociaciones y un grupo focal con los recicladores de la asociación Pichacay, a la cual, se tuvo acceso. La evaluación se hizo por medio de ocho preguntas semiestructuradas, que se muestran a continuación.

¿Sigue en la misma Asociación en la que estaba cuando empezó este proyecto?

El $4 \%$ se retiraron de las asociaciones a las que pertenecían al momento de iniciar el proyecto; mientras que, el resto continúan en las mismas asociaciones de recicladores; lo que se podría inferir que, de alguna manera, las intervenciones realizadas aportaron a mejorar las relaciones interpersonales, desarrollar una comunicación efectiva y motivación laboral, lo que contribuye a la estabilidad y permanencia en una organización.

¿Qué tan probable es que pueda poner en práctica lo que aprendió en los talleres?

Los resultados reflejan que el $72.41 \%$ del total de la población considera "muy probable" poner en práctica lo aprendido en los talleres; de hecho, ya lo han venido haciendo en su día a día. El $20.69 \%$ considera que es 〈probables que apliquen lo aprendido; mientras que, el 3,45\%, manifestó que es ‘algo probables que utilice los conocimientos adquiridos en los talleres; es decir, se puede evidenciar que estas personas sienten que en su labor diaria, los conocimientos y competencias adquiridas, pueden ser puestas en práctica con resultados satisfactorios, ya sea para solucionar problemas o para llegar a una mayor comprensión y empatía con las personas de su entorno, en el ámbito personal y laboral.

Indique tres cosas que haya aprendido en los talleres y que ha puesto en práctica en la asociación.

Los puntos que más se mencionan son: el compañerismo, el trabajo en equipo, la comunicación efectiva, el compromiso y la empatía, lo que da como resultado, mejores relaciones interpersonales en los equipos de trabajo, una mayor responsabilidad en el desarrollo de sus tareas como 
aporte al logro de metas grupales y más apoyo y comprensión entre compañeros, tanto en el buen desempeño, como en aquellas circunstancias personales por las que atraviesa cada uno de ellos. Así mismo, las personas mencionaron que sienten una mayor autoestima y el respeto a sí mismo, lo que les posibilita identificar sus propias necesidades y trabajar en sus satisfacciones.

¿Cree usted que lo que aprendió en los talleres ha ayudado a mejorar algo en la asociación en la que trabaja? Si contestó Sí, dé un ejemplo de lo que ha mejorado en la asociación gracias a los talleres.

El $89.66 \%$ de los participantes respondieron que sí, ya que gracias a las herramientas adquiridas, han podido mejorar la relación entre compañeros, la comunicación efectiva, la unión y compromiso entre ellos. Mencionan que se ha evidenciado un avance dentro de las asociaciones, debido a que ha mejorado la convivencia, que hacen referencia a la manera en la que cada uno interactúa con los demás miembros de su entorno. A su vez, manifiestan que ahora existe mayor apoyo mutuo entre ellos y han aprendido a comunicarse de mejor forma, lo que se evidencia en un trabajo más organizado y eficiente. Como ejemplo, ya no les toma tanto tiempo distribuir su trabajo de recolección, clasificación y embalaje de materiales reciclables. Por otra parte, gracias a las intervenciones han desarrollado su capacidad de autocontrol, lo que les permite reconocer y modificar su estado de ánimo, sus conductas y la forma de exteriorizar sus emociones para evitar disgustos y malos entendidos dentro de sus labores. La percepción es que los conflictos se han reducido. Finalmente, algunas personas mencionan, también, que han podido mejorar su capacidad de tomar decisiones y delegar funciones, de tal forma que se hace más sencillo alcanzar las metas a nivel de asociación, convirtiéndose en un equipo productivo y eficiente. 
Las actividades que se realizaron dentro de los talleres, por ejemplo, botar el huevo desde una altura procurando que no se rompa, contar experiencias personales, ver videos explicativos, ¿fueron útiles para su aprendizaje? ¿Por qué?

El 100\% indicó que las actividades realizadas durante los talleres fueron útiles para su aprendizaje debido a que ahora conocen la forma en la que se debe trabajar en equipo, a través de la solidaridad entre compañeros; llevaron a cabo una comunicación adecuada en el momento oportuno, el respeto a los demás y a las condiciones o circunstancia por las que está atravesando cada persona, el manejo de conflictos a través del diálogo y la empatía, la identificación de los propios errores y las oportunidades de mejora, la confianza en sí mismo y la autoestima; todo esto, les ayuda a formar un equipo más productivo, que logre alcanzar resultados positivos que sean de provecho para los integrantes de cada asociación.

Recuerdan permanentemente las actividades vivenciadas y ahora las toman como ejemplos cuando surge algún conflicto, pues, son capaces de encontrar otras alternativas más eficientes a los inconvenientes que se presentan.

\section{¿Considera que faltó algo en los talleres para mejorar el aprendizaje?}

Si contesta Sí, indique qué hizo falta.

El $79,31 \%$ de las personas mencionaron que los talleres estuvieron completos y que los temas abordados fueron de gran utilidad tanto para su trabajo como para su vida personal; mencionaron también que la diversidad en las actividades y la forma de impartir los conocimientos fueron dinámicos, lo que despertó el interés de los participantes, logrando una mejor asimilación de los conocimientos. Por otra parte, el 20,69\% indicó que faltó considerar aspectos como la limitación en el tiempo, ya que se puede profundizar más en los temas tratados para lograr mejores resultados; también mencionaron que faltaron actividades que les permitan integrarse entre asociaciones, ya que si bien se pudo trabajar como equipo en cada asociación, sin embargo, en una próxima ocasión, se pudiera aprovechar la diversidad y los aportes de los demás equipos como una estrategia de mejora conjunta. 
¿Qué recomendaciones o sugerencias tiene para los talleres que recibió?

El 31,03\% de las personas indicó que no tienen ninguna recomendación en cuanto al desarrollo de estos talleres, ya que se encuentran conformes tanto con la metodología como los temas abordados y los resultados obtenidos.

El resto de personas, es decir, el 68,97\% del total de participantes, indicó que aceptan como válidos estos talleres y recomiendan que se sigan impartiendo, ya que son beneficiosos para las personas. Recomiendan, también, que se generen nuevos espacios en los cuales se pueda profundizar en los temas que se trabajaron en estos talleres. Al parecer, el tiempo fue limitado para abordar todas sus pretensiones.

Así mismo, recomiendan que exista una mejor coordinación con la EMAC para que se pueda integrar a otras personas que también necesitan estas herramientas cognitivas y que no formaron parte de los talleres.

Recomendaría estos talleres a otros compañeros recicladores independientes (que no pertenecen a las asociaciones). ¿Por qué?

Todos respondieron que sí recomendarían estos talleres a los otros compañeros recicladores ya que, pudieron adquirir conocimientos y herramientas útiles para su trabajo, lo que les permite mejorar en aspectos personales y profesionales; a su vez, les sirve para alcanzar mejores resultados como asociación. 


\section{Conclusiones}

Se constató que los recicladores asociados de la ciudad de Cuenca laboran bajo condiciones difíciles y precarias, lo cual, facilita la proliferación de conflictos interpersonales. Las condiciones de vulnerabilidad de los recicladores los exponen a riesgos de carácter psicosocial que conlleva dificultades para alcanzar el bienestar personal y, mediante este, la armonía colectiva. Como lo menciona en la investigación de Martínez, Totterdell, Alcover \& Holman (2007), la regulación emocional es un proceso relevante para explicar cómo las demandas emocionales del trabajo están relacionadas con el agotamiento emocional. Es claro que los recicladores realizan una importante labor para la ciudad y para el ecosistema, por lo que esta ocupación debe ser alimentada por la academia, proporcionándoles las herramientas psicológicas que les permita aumentar su autoestima y, a la vez, desarrollar habilidades sociales para el mejor interrelacionamiento dentro de cada asociación. Para el desarrollo de estas habilidades fue importante potenciar ejercicios de carácter grupal, lúdico, que genere mayor involucramiento y fomenten el trabajo en equipo y colaborativo, incentivando la comunicación asertiva (Silva, 2013); todo esto encaminado a la mejora de la productividad, sin perder de vista el bienestar personal y colectivo. 
En este último aspecto, los recicladores cumplieron con el objetivo de desarrollar sus competencias intrapersonales, interpersonales y organizacionales, mediante las actividades planificadas por la Universidad del Azuay. Hubo gran participación y compromiso, lo que se evidenció en el alto índice de asistencia a las jornadas de trabajo, así como el entusiasmo demostrado en cada una de las actividades. Como lo refiere el estudio de Peña, Máiquez y Rodrigo (2013), la inclusión de contenidos de desarrollo personal en los programas de padres dirigidos a familias en riesgo psicosocial, puede frenar las influencias negativas que las situaciones estresantes que viven estas familias. Luego de esta participación, los recicladores cuentan con más herramientas que les permiten mejorar sus formas de comunicación, sus relaciones interpersonales, su organización y estructura, el trabajo en equipo y, como eje transversal, la actitud positiva hacia la vida.

Se hizo énfasis en la comunicación, debido a que se la considera como una habilidad básica para la vida y tiene que ver con la capacidad de expresarse verbal y preverbal en forma apropiada a la cultura y a las situaciones (Corrales, Quijano, \& Góngora, 2017). Se considera que la asertividad es una conducta y no tanto una característica de la personalidad, por lo que se habla de asertividad como una competencia en el campo de las habilidades sociales. De ahí que es posible realizar un entrenamiento de la autoafirmación, que permita mejorar las habilidades sociales de las personas (Naranjo, 2008).

A pesar de que este proyecto estuvo dirigido a los recicladores asociados, por diversas razones se tuvo una pequeña asistencia de recicladores independientes, sin embargo se los incluyó a todos en los talleres debido a que estas jornadas buscaban fortalecer las capacidades personales e institucionales de quienes asistían, por lo cual, no se podía negar la participación de quienes llegaban en busca de esta experiencia. Sería importante plantear una segunda intervención con este grupo de recicladores independientes (no asociados) y que también tengan la oportunidad de desarrollar sus competencias intra e interpersonales.

Algo muy importante que se llevan los recicladores, a más de los conocimientos y las experiencias vividas, es el certificado de haber participado en este programa de capacitación con el aval de la Universidad del Azuay y de la Empresa Pública Municipal de Aseo de Cuenca - EMAC. 
Es necesario mencionar que los participantes se mostraron muy satisfechos ante los resultados obtenidos al finalizar los talleres, dado que lo aprendido lo han puesto en práctica, lo que se evidencia en las evaluaciones de impacto realizadas. Es notorio el cambio de actitud hacia sí mismos y hacia sus asociaciones.

Como recomendaciones para las asociaciones, se puede mencionar el liderazgo, ya que las estructuras organizacionales no están correctamente definidas, lo que dificulta la comunicación y muchas veces causa confusión y tergiversación de la información de interés. En toda la intervención realizada, los grupos concluyeron que, para mantener buenas relaciones sociales y, por ende, incrementar su motivación y productividad, es necesario un liderazgo que inspire confianza y compromiso para el grupo, así como los haga partícipes a todos de las decisiones que se tomen, sea que puedan afectar positiva o negativamente a las asociaciones.

Es importante contar con líderes que gocen del respaldo de los miembros de las asociaciones, así como de los entes gubernamentales, para que sirvan de gestores de las necesidades y requerimientos de las personas y así puedan tratar de manera orgánica con las instituciones competentes. A su vez es fundamental, a más de establecer un canal, implementar un método de comunicación más efectivo, el cual, permita que los mensajes entre asociaciones e instituciones lleguen de manera oportuna y no se generen malos entendidos o retrasos en los eventos planificados.

Ya que se han mejorado los canales y métodos de comunicación, se debería aprovechar este escenario positivo para generar círculos de calidad en las asociaciones, con la finalidad de evaluar permanentemente, detectar errores o cuellos de botella y contribuir a la mejora continua de sus procesos organizacionales. Asimismo, sería un espacio para el diálogo constructivo y el fortalecimiento de capacidades interpersonales. 


\section{Referencias}

Balcazar, F. (2003). Investigación acción participativa: aspectos conceptuales y dificultades de implementación. Fundamentos en humanidades, 59-77.

Campbell, L., Campbell, B., \& Dickinson, D. (2002). Inteligencias múltiples. Buenos Aires: Troquel

Campos, J., Dahl, A., Walle, E., \& Main, A. (2011). Reconceptualizing Emotion Regulation. Emotion Review, 26-35.

Constituyente, A. N. (2008). Constitución de la República del Ecuador. Montecristi.

Cornejo, M., \& Tapia, M. (2012). Redes sociales y relaciones interpersonales en internet. Fundamentos en humanidades, 219-229.

Corrales, A., Quijano, N., \& Góngora, E. (2017). Empatía, comunicación asertiva y seguimiento de normas. Un programa para desarrollar habilidades para la vida. Enseñanza e investigación en Psicología, 58-65.

Diaz-Cano, M. \&.-V. (2018). Consolidación organizativa de los recicladores. Estudios del desarrollo humano y socioambiental, 41-87.

Estrada, L. (2018). Motivación y emoción. Bogotá: Fundación Universitaria del Área Andina.

Fernández, P., \& Extremera, N. (2005). La inteligencia emocional y la educación de las emociones desde el modelo de Mayer y Salovey. Revista universitaria de formación del profesorado, 63-93.

Freitas-Magalhaes, A. (2007). La psicología de las emociones: la fascinación del rostro humano. Porto: FEELab Science Books.

Gagne, M., \& Deci, E. (2005). Self-determination theory and work motivation. Journal of Organizational Behavior, 331-362.

Kawamoto, T., Ura, M., \& Nittono, H. (2015). Intrapersonal and interpersonal processes of social exclusion. Frontiers in neuroscience, $1-11$. 
Levenson, R. (1999). The intrapersonal functions of emotion. Cognition and emotion, 481-504.

Martínez, Í., Totterdell, P., Alcover, C., \& Holman, D. (2007). Emotional labour and emotional exhaustion: Interpersonal and intrapersonal mechanisms. Work \& Stress, 30-47.

McClelland, D. (1973). Evaluar la competencia en lugar de la inteligencia. American Psychologist, 1-14.

Nagles, N. (2005). El desarrollo de competencias. Revista Escuela de Administración de Negocios, 99-114.

Naranjo, M. L. (2007). Autoestima: un factor relevante en la vida de la persona y tema esencial del proceso educativo. Actualidades investigativas en educación, 1-27.

Naranjo, M. L. (2008). Relaciones interpersonales adecuadas mediante una comunicación y conducta asertivas. Actualidades investigativas en educación, 1-27.

Naranjo, M. L. (2011). Perspectivas sobre la comunicación. Actualidades investigativas en educación, 1-32.

Peña, M., Máiquez, M., \& Rodrigo, M. (2013). Efectos de la inclusión de contenidos de desarrollo personal en un programa de educación parental para familias en riesgo psicosocial. Anales de psicología, 201-210.

PNUD. (23 de Junio de 2020). PNUD Ecuador. PNUD Ecuador: https:// www.ec.undp.org/content/ecuador/es/home/sustainable-development-goals/goal-3-good-health-and-well-being.html

Rodríguez, E. (2019). La importancia de la comunicación asertiva. Clic, $1-2$.

Salavera, C., \& Usán, P. (2018). Adaptación del Cuestionario de Competencia Interpersonal ICQ-15 con Población Adolescente Hispanohablante. Revista Iberoamericana de Diagnóstico y Evaluación Psicológica, 29-39.

Sánchez, J., Magdaniel, Y., \& Sánchez, I. (2013). Comunicación asertiva en empresas recicladoras de plástico. Forum humanes, 1-11. 
Sánchez-Vidal, A. (2017). Empoderamiento, liberación y desarrollo humano. Psychosocial Intervention, 155-163.

SENPLADES. (2017). Plan Nacional para el Buen Vivir 2017-2021. Quito.

Silva, M. (2013). Habilidades blandas, fundamentales para el desarrollo personal. Revista Educar, 22-23.

Steers, R., \& Mowday, R. (2004). The future of work motivation theory. Academy of Management Review, 379-387.

Suárez, I., \& Mendoza, B. (2008). Desarrollo de la inteligencia emocional y de la capacidad para establecer relaciones interpersonales e intrapersonales. Laurus, 76-95.

Velenturf, A., \& Jopson, J. (2019). Haciendo el caso de negocios para la recuperación de recursos. Ciencia del medio ambiente total, 1031-1041.

Weigner, B. (1992). Motivación humana: metáforas, teorías e investigaciones. California: Sage publications.

Werkuyten, M., \& Nekuee, S. (2001). Selfesteem, discrimination, and coping among refugees: the moderating role of self-categorization. Applied Social Psychology, 1058-1075.

Yánez, R., Arenas, M., \& Ripoll, M. (2010). El impacto de las relaciones interpersonales en la satisfacción laboral general. Liberabit, 193201. 